\title{
Dynamics of a gravity car race with application to the Pinewood Derby
}

\author{
B. P. Mann, M. M. Gibbs, and S. M. Sah \\ Mechanical Engineering and Material Science Department, Duke University, Durham, NC 27708, USA
}

Correspondence to: B. P. Mann (brian.mann@duke.edu)

Received: 18 August 2012 - Revised: 17 November 2012 - Accepted: 23 November 2012 - Published: 4 December 2012

Abstract. This paper investigates the underlying physics of a gravity car race. This work seeks to provide a sound theoretical basis to elucidate the design considerations that maximize performance while simultaneously dispelling false assertions that may arise from incomplete analyses. The governing equations are derived and solved analytically to predict race times; trend analyses are then performed along with a sensitivity analysis to ascertain the most important factors that influence performance. The inferences from a conservative energy balance are then compared with the predictions from the full set of differential equations, which include the dissipative terms associated with air resistance and friction.

\section{Introduction}

Gravity car races have provided their enthusiast numerous thrills over the years. While some of the longer-standing competitions include the Soap Box Derby and the Pinewood Derby, new events, such as the Extreme Gravity Racing Series and the Wile Street-luge Sliders of the X-Games, have also recently emerged. Although the race vehicles from these events can drastically vary in their size, shape, and complexity, they also share many common challenges. For example, they are all driven by the force of gravity and must minimize the forces that oppose the vehicle motions, such as wind resistance and friction.

The pinewood derby is one of the more distinctive events. It originated as a Cub Scout competition where elementary school children raced a car assembled from a kit consisting of a block of wood, four nails and wheels. A small industry has sprung up around the pinewood derby, with countless internet sites and books offering tips, tricks and even enhanced car parts to the estimated 43 million children that have built pinewood derby cars since its founding (Garguilo and Garguilo, 2011; Pedigo, 2002; Reinke, 2010). While there are many who offer advice, only a few scientific investigations have been published and it is rare to find accurate explanations on how certain modifications could result in faster race times (Coletta and Evans, 2008). In reference Coletta and
Evans (2008), Coletta and Evans used an algebraic function to obtain an analytical expression for the time and speed as function of the distance traveled along the track. Their analysis included the rotational energy of the wheels, rolling friction, and air resistance. In an early work, Cowley et al. (1989) obtained an approximation for the race time by considering the curved section of the track as two straight parts.

This paper seeks to provide a sound theoretical basis for making car modifications from the derivations herein. Trends in the peak velocity and race time are investigated from an energy balance and the governing equations. In addition, we compare the results of our analyses to a series of experimental results that verify the trends unveiled in our analyses also occur experimentally. As a part of our theoretical investigations, a sensitivity analysis was performed to ascertain the relative importance of five key parameters on reducing race times.

The work contained in this paper is organized as follows. The next section considers the conservative system and then performs an energy balance to derive an expression for the peak velocity. In Sect. 3, the equation of motions are derived for the two sections of the track, namely the straight and curved regions, taking into account the nonconservative forces. Analytical solutions are then derived for the governing equations. A sensitivity analysis is performed to determine the relative importance of altering the cross sectional 

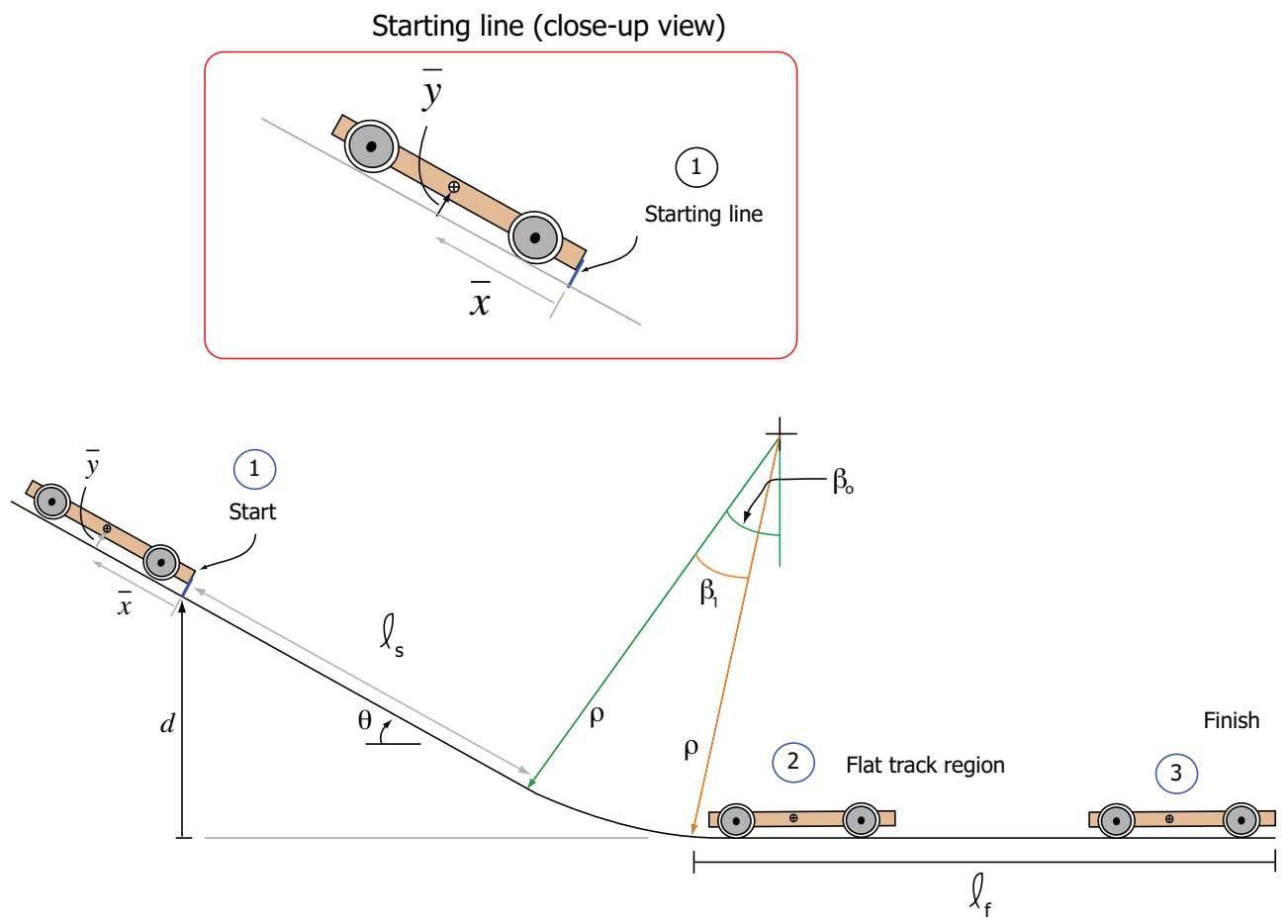

Figure 1. Illustration of a car at the starting line (upper left) with the location of the center of mass marked by $\bar{x}$ and $\bar{y}$. Bottom illustration shows the car at several locations along the track and the important track geometry.

area, car mass, friction coefficient, and wheel parameters to reduce the race times. The trends from the theoretical investigations are then compared with a series of experimental tests in Sect. 4. Finally, the last section provides a discussion of the combined theoretical and experimental investigations. The discussion also explains several additional opportunities for improving performance beyond the conclusions made from theoretical and experimental investigations.

\section{Conservative system energy balance}

An energy balance often provides a useful alternative to directly solving the governing differential equations and is used here to elucidate how design changes can influence the vehicle performance. The generic form of an energy balance is

$\mathcal{T}_{1 \rightarrow 2}+\mathcal{U}_{1 \rightarrow 2}=\mathcal{W}_{\mathrm{a}}-\mathcal{W}_{\mathrm{d}}$

where $\mathcal{T}_{1 \rightarrow 2}$ is the change in kinetic energy, $\mathcal{U}_{1 \rightarrow 2}$ is the change in potential energy, $\mathcal{W}_{\mathrm{a}}$ is the added work of external forces, and $\mathcal{W}_{\mathrm{d}}$ is the work due to energy dissipation over the time interval from $t_{1}$ to $t_{2}$. Figure 1 shows the car at three locations along the track, i.e. the starting line, at the entry into the final horizontal section, and at the finish; this section will use these three locations to gain insight when applying an energy balance. Consider first the transition from the starting line (location one) to the beginning of the final straigth section (location two). When the system is treated as a rigid body, as opposed to the point mass assumption of Jobe (2004), both translational and rotational energy terms appear in the kinetic energy

$\mathcal{T}_{1 \rightarrow 2}=\frac{1}{2} m v^{2}+\frac{N}{2}\left(I_{w} \dot{\phi}^{2}\right)$,

where $m$ is the total vehicle mass, $v$ is the velocity of the center of mass, and $N$ is the number of wheels, with a mass moment of intertia $I_{w}$, which rotate with an angular velocity $\dot{\phi}$. We next assume the wheels roll without slip, which allows the car's velocity to be written in terms of the angular rotations of the wheels $v=r_{o} \dot{\phi}$, where $r_{o}$ is the wheel radius. After definining the wheel mass moment of inertia in terms of a radius of gyration, the change in kinetic energy can be written as

$\mathcal{T}_{1 \rightarrow 2}=\frac{1}{2} m\left(1+N \frac{m_{o}}{m}\left(\frac{k}{r_{o}}\right)^{2}\right) v^{2}$,

where $m_{o}$ is the mass and $k$ the radius of gyration of a single wheel. The change in potential energy between the two locations is given by

$\mathcal{U}_{1 \rightarrow 2}=m g(\bar{y}(1-\cos \theta)-\bar{x} \sin \theta-d)$,

where $d$ is the vertical distance to the starting position on the track, $\bar{y}$ is the vertical distance from the track to the center of mass, $\bar{x}$ is the horizontal distance from the front of the car 

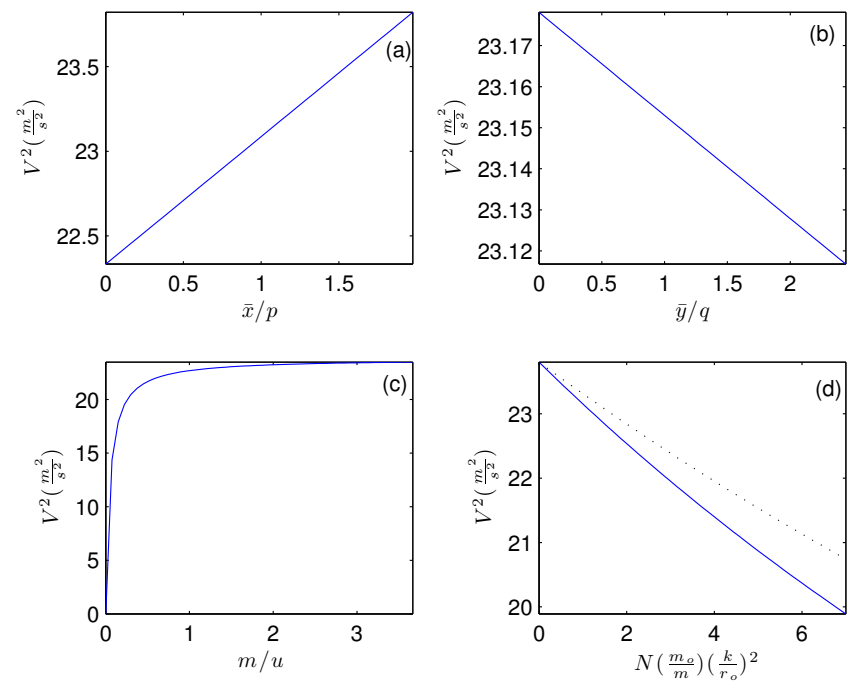

Figure 2. Energy balance trends showing the trends in $v^{2}$ while varying $\bar{x}$ (a), $\bar{y}$ (b), $m$ (c), and wheel parameters (d). The dotted line in (d) represents a car with three wheels contacting the track or $N=3$. The parameters listed in Table 1 and the normalization constants $p=0.09 \mathrm{~m}, q=0.013 \mathrm{~m}$, and $u=0.1091 \mathrm{Kg}$ were used.

to the center of mass, and $\theta$ is angle of incline of the track (see Fig. 1 for geometry). Assuming no work is added or dissipated from the system, Eqs. (3) and (4) can be inserted into Eq. (1) to obtain an expression for the velocity

$v^{2}=\frac{2 g(d+\bar{x} \sin \theta+\bar{y}(\cos \theta-1))}{1+N \frac{m_{o}}{m}\left(\frac{k}{r_{o}}\right)^{2}}$.

This energy balance solution alone can be used to provide much insight into how design changes will influence the vehicle velocity. More specifically, $v^{2}$ will increase linearly for linear changes in $\bar{x}$, but $v^{2}$ will decrease linearly for linear changes in $\bar{y}$; this suggests the center of mass should be located as far back and close to the track as possible. Focusing on the denominator of Eq. (5), the combined terms $N \frac{m_{o}}{m}\left(\frac{k}{r_{o}}\right)^{2}$ increase the denominator to be greater than one; thus the smaller this grouping of terms can be made, the greater the increase in $v^{2}$. Setting $m_{o}$ equal to zero in this expression gives the same result as if the car was being modeled as a point mass. This assumption, which is commonly made in pinewood derby analyses, neglects the reduction in race time that can be achieved by manipulating the combined terms shown in Fig. 2d.

To illustrate the importance of $\bar{x}$ and $\bar{y}$, along with the terms that appear in the denominator, we have varied the different model parameters to ascertain their influence on $v^{2}$. For example, Fig. 2 shows the trends in the car's peak velocity for changes in the location of the center of mass and wheel parameters - at least in the absence of any dissipative forces. In these plots, we have normalized the horizontal axes
Table 1. Parameters used for energy balance trend analysis.

\begin{tabular}{ll}
\hline Parameter & Value \\
\hline$\theta$ & $26^{\circ}$ \\
$d$ & $1.17(\mathrm{~m})$ \\
$\bar{x}$ & $98(\mathrm{~mm})$ \\
$\bar{y}$ & $13(\mathrm{~mm})$ \\
$N$ & 4 \\
$g$ & $9.81\left(\mathrm{~m} \mathrm{~s}^{-2}\right)$ \\
$m$ & $0.191(\mathrm{Kg})$ \\
$m_{o}$ & $2.65 \times 10^{-3}(\mathrm{Kg})$ \\
$r_{o}$ & $1.51\left(\mathrm{~mm}^{2}\right)$ \\
$k$ & $10.7\left(\mathrm{~mm}^{-7}\left(\mathrm{Kg} \mathrm{m}^{-2}\right)\right.$ \\
$I_{w}$ & $3.06 \times 10^{-7}$ \\
\hline
\end{tabular}

by the parameters of an out-of-the-box pinewood derby car and restricted the range to attainable limits. After applying the track geometry, parameters given in Table 1, we observe $v^{2}$ to increase and decrease linearly with changes in $\bar{x}$ and $\bar{y}$ respectively, with $\bar{x}$ having nearly four times the impact on $v^{2}$ than $\bar{y}$. However, the total mass of the car and the combined terms that appear in the denominator dominate the expression for $v^{2}-$ increasing the mass to double the out-of-box parameter has more than 15 times the impact of doing the same to $\bar{x}$. The $v^{2}$ trend in plot (d) highlights the benefit of reducing the $m_{o} / m$ ratio, which can be accomplished by either removing mass from the wheels or by increasing the car mass relative to the wheel mass. This plot additionally shows that substantial increases could be achieved in the peak velocity provided it were possible to reduce the mass moment of inertia for the wheels, i.e. this is captured by a reduction in the radius of gyration $k$. Thus, we highlight that removing material from the outer portion of the wheel would reduce both $m_{o}$ and $k$ and should have a double effect to increase the peak velocity.

In summary, we have presented an energy balance that suggests $\bar{y}$ should be as small as possible and $\bar{x}$ and $m$ should be as large as possible. However, it is important to recognize certain practical and physical restrictions that also constrain these values; for example, locating $\bar{x}$ behind the rear axle would cause the car to tip over, so the rear axle should be located as far back as possible and $\bar{x}$ should be just in front of the rear axle. Similarly, $\bar{y}$ must provide clearance between the car bottom and the raised center of the track. While Fig. 2 also suggests that lifting one wheel off the track will increase the car's velocity, additional energy losses occur if the fourth wheel is not low enough to contact the raised center of the track, which helps to maintain alignment. The energy balance neglects dissipative forces and therefore $v^{2}$ continues to grow as mass is added to the car; this trend does not reflect the fact that after a certain point velocity reduction due to friction will outweigh the benefit of adding additional mass. We therefore consider the results of this section as guidelines with certain limits and explore this notion even further 
in the upcoming sections. In the next section, the equations of motion are derived with the inclusion of the nonconservative forces.

\section{Equations of motion and their analyses}

This section derives the equation of motion for a prototypical pinewood derby car. The governing equations are later used to further explore the influence of design choices and parameter uncertainty on the race times of the vehicle. The derivation that follows has been split into two parts with separate derivations for the curved and straight sections of the track. To complete the analysis, it was assumed that the car wheels would roll without slip and that the length of the car is negligible compared to the length of the track.

\subsection{Straight track sections}

Consider the inclined portion of the track shown in Fig. 1. Applying notations from the previous sections, the kinetic energy becomes

$\mathcal{T}=\frac{1}{2} m\left(1+N \frac{m_{o}}{m}\left(\frac{k}{r_{o}}\right)^{2}\right) \dot{s}^{2}$,

where $s$ is the distance the car's center of mass has traveled along a straight section of the track and $\dot{s}=v$ is the vehicle's velocity along the flat section of the track. The potential energy of the system is given by

$\mathcal{U}=m g(d+\bar{x} \sin \theta+\bar{y} \cos \theta-s \sin \theta)$.

In addition to the conservative forces, the nonconservative forces of air resistance and sliding friction between the wheels and axles must be taken into account. Here, we note sliding friction causes a moment that opposes the wheel rotation. Applying Lagrange's equation, where the nonconservative forces from drag and friction are included, results in the following governing equation:

$m\left[1+N \frac{m_{o}}{m}\left(\frac{k}{r_{o}}\right)^{2}\right] \ddot{s}-m g \sin \theta=-\frac{1}{2} \rho_{\mathrm{a}} A C_{\mathrm{D}} \dot{s}|\dot{s}|-2 f_{\mathrm{A}} \frac{r_{i}}{r_{o}}-2 f_{\mathrm{B}} \frac{r_{i}}{r_{o}}$,

where $f_{\mathrm{A}}$ is the sliding friction force on a front wheel and $f_{\mathrm{B}}$ is the sliding friction force on a back wheel, $\rho_{\mathrm{a}}$ is the air density, $C_{\mathrm{D}}$ the drag coefficient, and $A$ is the projected crosssectional area. To derive the expression for the nonconservative forces, we note that a roll with slip condition was applied. To resolve the frictional forces $f_{\mathrm{A}}$ and $f_{\mathrm{B}}$, a Coulomb friction law was applied to write $f_{\mathrm{A}}=\mu_{\mathrm{k}} N_{\mathrm{A}}$ and $f_{\mathrm{B}}=\mu_{\mathrm{k}} N_{\mathrm{B}}$, where $\mu_{\mathrm{k}}$ is the kinetic coefficient of friction and $N_{\mathrm{A}}$ and $N_{\mathrm{B}}$ are the normal forces that act at the locations shown in Fig. 3; next, the moments were summed about the rear axle to obtain expression for the normal forces

$N_{\mathrm{A}}=\left(m-4 m_{o}\right) \frac{\left(h_{o}-\bar{y}_{\mathrm{c}}\right)(\ddot{s}-g \sin \theta)+\left(\ell_{\mathrm{B}}-\bar{x}_{\mathrm{c}}\right) g \cos \theta}{\ell_{\mathrm{B}}-\ell_{\mathrm{A}}}$
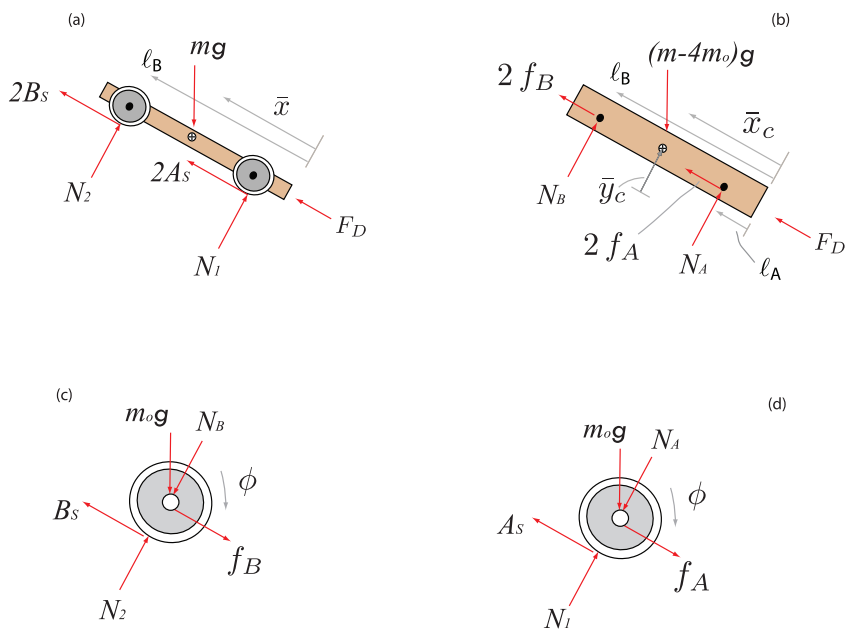

Figure 3. Free body diagram of the forces on (a) the assembled car, (b) only the car body, (c) the rear wheel, and (d) the front wheel.

summing the moments about an axis through $A$ and solving for $N_{\mathrm{B}}$ in a similar manner gives

$N_{\mathrm{B}}=\left(m-4 m_{o}\right) \frac{\left(\bar{y}_{\mathrm{c}}-h_{o}\right)(\ddot{s}-g \sin \theta)-\left(\ell_{\mathrm{A}}-\bar{x}_{\mathrm{c}}\right) g \cos \theta}{\ell_{\mathrm{B}}-\ell_{\mathrm{A}}}$

where $\ell_{\mathrm{A}}$ is the distance from the front of the car to the front axle, $\ell_{\mathrm{B}}$ is the distance from the front of the car to the rear axle, and $\bar{x}_{\mathrm{c}}$ is the center of mass location for the car body (see Fig. 1). After inserting the expressions for $f_{\mathrm{A}}=\mu_{\mathrm{k}} N_{\mathrm{A}}$ and $f_{b}=\mu_{\mathrm{k}} N_{\mathrm{B}}$ into Eq. (8), the governing equation takes the form

$\ddot{s}+\gamma \dot{s}|\dot{s}|=\eta$

where the parameters $\gamma$ and $\eta$ are given by

$$
\begin{aligned}
& \gamma=\frac{\rho_{\mathrm{a}} A C_{\mathrm{D}}}{2 m\left(1+N \frac{m_{o}}{m}\left(\frac{k}{r_{o}}\right)^{2}\right)}, \\
& \eta=\frac{\sin \theta-2 \mu_{\mathrm{k}} \frac{r_{i}}{r_{o}}\left(1-4 \frac{m_{o}}{m}\right) \cos \theta}{1+N \frac{m_{o}}{m}\left(\frac{k}{r_{o}}\right)^{2}} g .
\end{aligned}
$$

Before departing this section, we note the general form of Eq. (11) can be applied to either the horizontal $(\theta=0)$ or inclined $(\theta \neq 0)$ sections of the track.

\subsubsection{Analytical solution for the straight track sections}

This section derives an exact analytical solution for the straight sections of the track. Along the straight track the sign of the car's velocity is always positive therefore $|\dot{s}|=\dot{s}$ in Eq. (11). The analysis starts by substituting $v=\dot{s}$ into Eq. (11) to obtain

$\dot{v}+\gamma v^{2}=\eta$ 
where $v$ is the vehicle's velocity. Here, we note the value of $\eta$ in Eq. (13) can be either positive or negative depending on which part of the straight track the car is located. When the car is on the inclined section $\eta$ is positive, but it takes on a negative value for the horizontal section of the track. Therefore the solution to Eq. (13) must consider the two parts of the straight track. In the case of the inclined track we begin by rearranging Eq. (13) to give the following differential relationship:

$\frac{\mathrm{d} v}{\eta-\gamma v^{2}}=\mathrm{d} t$

After integrating this relationship and solving for $v$, the analytical expression for the vehicle's velocity becomes

$v=\sqrt{\frac{\eta}{\gamma}} \tanh \left(\sqrt{\gamma \eta} t+\tanh ^{-1}\left(\sqrt{\frac{\gamma}{\eta}} v_{0}\right)\right)$,

where $v_{0}=v(0)$ is the initial velocity on the inclined track. To obtain the analytical expression for the car's position, Eq. (15) was integrated to obtain

$$
\begin{aligned}
s= & s_{o}+\frac{1}{\gamma} \ln \left[\cosh \left(\sqrt{\gamma \eta} t+\tanh ^{-1}\left(\sqrt{\frac{\gamma}{\eta}} v_{0}\right)\right)\right]-\frac{1}{\gamma} \\
& \ln \left[\cosh \left(\tanh ^{-1}\left(\sqrt{\frac{\gamma}{\eta}} v_{0}\right)\right)\right],
\end{aligned}
$$

where $s_{o}$ is the initial position of the vehicle. Assuming the car starts from rest, where $s_{o}=v_{o}=0$, the analytical solutions for the velocity and position can be simplified to

$v=\sqrt{\frac{\eta}{\gamma}} \tanh (\sqrt{\gamma \eta} t)$,

$s=\frac{1}{\gamma} \ln (\cosh (\sqrt{\gamma \eta} t))$.

We next consider the horizontal section of the track and presume the car enters this section at $t=t_{1}$. The solution to Eq. (13) is given by

$v=-\sqrt{-\frac{\eta}{\gamma}} \tan \left(\sqrt{-\gamma \eta}\left(t-t_{1}\right)-\tan ^{-1}\left(\sqrt{\frac{-\gamma}{\eta}} v_{1}\right)\right)$,

where $v_{1}=v\left(t_{1}\right)$ is the initial velocity of the car as it enters the horizontal section of the track. The position of the car along the horizontal section of the track is obtained by integrating Eq. (18), which yields the following following expression:

$$
\begin{aligned}
u & =-\frac{1}{\gamma} \ln \left[\sec \left(-\sqrt{-\gamma \eta}\left(t-t_{1}\right)+\tan ^{-1}\left(\sqrt{\frac{-\gamma}{\eta}} v_{1}\right)\right)\right] \\
& -\frac{1}{\gamma} \ln \left[\sec \left(\tan ^{-1}\left(\sqrt{\frac{-\gamma}{\eta}} v_{1}\right)\right)\right],
\end{aligned}
$$

where $u$ is position along the horizontal section of the track. In deriving this result, it is important note that $u\left(t_{1}\right)=0$ was applied to obtain Eq. (19).

\subsection{Curved track equation of motion}

This section derives the governing equation for the curved section of the track and is followed by the development of an approximate analytical solution. We first express the kinetic energy of the system using the roll without slip condition and a radius of gyration description for the wheel mass moment of inertia, as in the previous expression for the kinetic energy, to obtain the following:

$\mathcal{T}=\frac{1}{2} m\left[(\rho-\bar{y})^{2}+N \frac{m_{o}}{m}\left(\frac{k}{r_{o}}\right)^{2} \rho^{2}+\frac{I_{G}}{m}\right] \dot{\beta}^{2}$,

where $\rho$ is the track radius of curvature, $I_{G}$ is the car's mass moment of inertia, and $\beta$ is the angular position of the center of mass. The potential energy of the system is given by

$\mathcal{U}=m g(\rho-\bar{y})(1-\cos \beta)$.

Applying Lagrange's equation, where the nonconservative forces from drag and friction are included, results in the following governing equation:

$$
\begin{gathered}
m\left[(\rho-\bar{y})^{2}+N \frac{m_{o}}{m}\left(\frac{k}{r_{o}}\right)^{2} \rho^{2}+\frac{I_{G}}{m}\right] \ddot{\beta}+m g(\rho-\bar{y}) \sin \beta \\
=-\frac{\rho_{\mathrm{a}}}{2} A C_{\mathrm{D}} \bar{\rho}^{3} \dot{\beta}|\dot{\beta}|-2 \mu_{\mathrm{k}} \rho \frac{r_{i}}{r_{o}}\left(N_{\mathrm{A}}+N_{\mathrm{B}}\right),
\end{gathered}
$$

To resolve the normal forces $N_{\mathrm{A}}$ and $N_{\mathrm{B}}$, the moments were summed about the front and rear axles, which yields

$$
\begin{gathered}
N_{\mathrm{A}}=\frac{m-4 m_{o}}{\ell_{\mathrm{B}}-\ell_{\mathrm{A}}}\left[\left(\ell_{\mathrm{B}}-\bar{x}_{\mathrm{c}}\right)\left(g \cos \beta+(\rho-\bar{y}) \dot{\beta}^{2}\right)\right. \\
\left.\left.+\left(r_{o}-\overline{y_{\mathrm{c}}}\right)(g \sin \beta+\ddot{\beta})(\rho-\bar{y})\right)\right]+\frac{I_{g} \ddot{\beta}}{\ell_{\mathrm{B}}-\ell_{\mathrm{A}}}, \\
N_{\mathrm{B}}=\frac{m-4 m_{o}}{\ell_{\mathrm{B}}-\ell_{\mathrm{A}}}\left[-\left(r_{o}-\overline{y_{\mathrm{c}}}\right)(g \sin \beta+(\rho-\bar{y}) \ddot{\beta})\right. \\
+\left(\bar{x}_{\mathrm{c}}-\ell_{\mathrm{A}}\right)\left(g \cos \beta+(\rho-\bar{y}) \dot{\beta}^{2}\right]-\frac{I_{G} \ddot{\beta}}{\ell_{\mathrm{B}}-\ell_{\mathrm{A}}},
\end{gathered}
$$

Equations (23)-(24) can then be combined to obtain the governing equation for the curved section of the track,

$\ddot{\beta}+\mu \dot{\beta}|\dot{\beta}|+\alpha \cos \beta+\omega^{2} \sin \beta=0$,

where the parameters $\mu, \omega^{2}, \alpha$ and $\tilde{m}$ are given by

$$
\begin{aligned}
\mu & =\frac{\left[\frac{\rho_{\mathrm{a}}}{2} A C_{\mathrm{D}} \bar{\rho}^{2}+2 \mu_{\mathrm{k}} \rho \frac{r_{i}}{r_{o}}\left(m-4 m_{o}\right)\right](\rho-\bar{y})}{\tilde{m}}, \\
\omega^{2} & =\frac{m g(\rho-\bar{y})}{\tilde{m}}, \\
\alpha & =\frac{2 \mu_{\mathrm{k}} \rho \frac{r_{i}}{r_{o}}\left(m-4 m_{w}\right) g}{\tilde{m}} \\
\tilde{m} & =m\left[(\rho-\bar{y})^{2}+N \frac{m}{m_{o}}\left(\frac{k}{r_{o}}\right)^{2} \rho^{2}+\frac{I_{G}}{m}\right] .
\end{aligned}
$$




\subsubsection{Approximate solution for the curved region}

In order to find an approximate solution, we Taylor expand Eq. (25) and introduce a small parameter $\epsilon$ which gives

$\ddot{\beta}+\epsilon \mu \dot{\beta}|\dot{\beta}|+w^{2} \beta+\epsilon c_{2} \beta^{2}+c_{1}=0$,

where

$c_{1}=\alpha, \quad c_{2}=\frac{-\alpha}{2}$.

The book-keeping parameter $\epsilon$ will serve as a perturbation parameter and will be set equal to unity at the end. In order to find an approximate solution to Eq. (27), we use the method of Krylov-Bogoliubov-Mitropolsky (KBM), see Mickens (1996) and Minorsky (1962). Equation (27) can be written as

$\ddot{\beta}+w^{2} \beta+c_{1}=\epsilon F(\beta, \dot{\beta})$,

where

$F(\beta, \dot{\beta})=-\mu \dot{\beta}|\dot{\beta}|-c_{2} \beta^{2}$.

We assume that the solution to Eq. (27) takes the following form

$\beta=a \cos \psi-\frac{c_{1}}{w^{2}}+\epsilon u_{1}(a, \psi)+\epsilon^{2} u_{2}(a, \psi)+\ldots$,

where the $u_{i}(a, \psi)$ are periodic functions of $\psi$, with period $2 \pi$, and the quantities $a$ and $\psi$ are functions of time defined by the following equations:

$\dot{a}=\epsilon A_{1}(a)+\epsilon^{2} A_{2}(a)+\ldots$,

$\dot{\psi}=w+\epsilon B_{1}(a)+\epsilon^{2} B_{2}(a)+\ldots$

The functions $u_{i}(a, \psi), A_{i}(a)$ and $B_{i}(a)$ are to be chosen in such a way that Eq. (31), after replacing $a$ and $\psi$ by the functions defined in Eqs. (32)-(33), is a solution to Eq. (27). Taking into account Eqs. (31), (32) and (33), the first derivative of $\beta$ takes the form

$$
\begin{aligned}
\dot{\beta} & =-a w \sin \psi+\epsilon\left(A_{1} \cos \psi-a B_{1} \sin \psi+w \frac{\partial u_{1}}{\partial \psi}\right) \\
& +\epsilon^{2}\left(A_{2} \cos \psi-a B_{2} \sin \psi+A_{1} \frac{\partial u_{1}}{\partial a}+B_{1} \frac{\partial u_{1}}{\partial \psi}+w \frac{\partial u_{2}}{\partial \psi}\right)+\ldots,
\end{aligned}
$$

On the other hand, the right-side of Eq. (29) can be rewritten to the form:

$\epsilon F(\beta, \dot{\beta})=\epsilon F\left(a \cos \psi-\frac{c_{1}}{w^{2}},-a w \sin \psi\right)+\epsilon^{2}\left[u_{1} F_{\beta}\left(a \cos \psi-\frac{c_{1}}{w^{2}},-a w \sin \psi\right)\right.$

$$
\left.+\left(A_{1} \cos \psi-a B_{1} \sin \psi+w \frac{\partial u_{1}}{\partial \psi}\right) F_{\dot{\theta}}\left(a \cos \psi-\frac{c_{1}}{w^{2}},-a w \sin \psi\right)\right] .
$$

Substituting Eqs. (31)-(35) into Eq. (29), collecting the terms with like powers of $\epsilon$, and setting them to zero, gives

$$
\begin{aligned}
& \frac{\partial^{2} u_{1}}{\partial \psi^{2}}+u_{1}=F_{0}(a, \psi)+2 A_{1} \sin \psi+2 a B_{1} \cos \psi, \\
& \frac{\partial^{2} u_{2}}{\partial \psi^{2}}+u_{2}=F_{1}(a, \psi)+2 A_{2} \sin \psi+2 a B_{2} \cos \psi .
\end{aligned}
$$

The functions $A_{i}(a), B_{i}(a)$, and $u_{i}(a, \psi)$ can be found by first expanding $F_{j}(a, \psi)$ and $u_{i}(a, \psi)$ into a Fourier series:

$$
\begin{gathered}
F_{j}=g_{j, 0}(a)+\sum_{n=1}^{\infty}\left[g_{j, n} \cos (n \psi)+h_{j, n} \sin (n \psi)\right], \\
u_{i}(a, \psi)=p_{i, 0}+\sum_{n=1}^{\infty}\left[p_{i, n} \cos (n \psi)+q_{i, n} \sin (n \psi)\right],
\end{gathered}
$$

where

$g_{j, n}=\frac{1}{\pi} \int_{0}^{2 \pi} F_{j}\left(a \cos \psi-\frac{c_{1}}{w^{2}},-a w \sin \psi\right) \cos (n \psi) d \psi$,

$h_{j, n}=\frac{1}{\pi} \int_{0}^{2 \pi} F_{j}\left(a \cos \psi-\frac{c_{1}}{w^{2}},-a w \sin \psi\right) \sin (n \psi) d \psi$,

and then equating the harmonics of the same order. It should be noted that the integration above is broken into two partsone with the limits 0 and $\pi$ and the other with the limits $\pi$ and $2 \pi$. For example,

$$
\begin{aligned}
h_{0,1} & =\frac{1}{\pi} \int_{0}^{2 \pi} F_{0}\left(a \cos \psi-\frac{c_{1}}{w^{2}},-a w \sin \psi\right) \sin (\psi) d \psi \\
& =\frac{1}{\pi} \int_{0}^{2 \pi}-\mu(-a w \sin \psi)|a w \sin \psi| \sin \psi-c_{2}\left(a \cos \psi-\frac{c_{1}}{w^{2}}\right)^{2} \sin \psi d \psi \\
& =\frac{a^{2} w^{2}}{\pi}\left[\int_{0}^{\pi} \sin ^{3} \psi d \psi-\int_{\pi}^{2 \pi} \sin ^{3} \psi d \psi\right]=\frac{8 a^{2} w^{2}}{3 \pi} .
\end{aligned}
$$

After doing the above calculations we found

$$
\begin{aligned}
\beta & =a \cos \psi-\frac{c_{1}}{w^{2}}+\epsilon\left[\frac{c_{1} c_{2} a}{w^{4}}-\frac{2 c_{1} c_{2} a}{3 w^{4}} \cos 2 \psi-\frac{c_{1} c_{2}}{4 w^{4}}\right. \\
& \left.\cos 3 \psi-\frac{8 \mu a^{2}}{9 \pi} \sin 2 \psi-\frac{\mu a^{2}}{3 \pi} \sin 3 \psi\right] \\
\dot{\beta} & =-a w \sin \psi+\epsilon\left[-\frac{4 w \mu a^{2}}{3 \pi} \cos \psi+\frac{c_{1} c_{2} a}{w^{3}} \sin \psi\right. \\
& +w\left(\frac{4 c_{1} c_{2} a}{3 w^{4}} \sin 2 \psi+\frac{3 c_{1} c_{2}}{4 w^{4}} \sin 3 \psi-\frac{16 \mu a^{2}}{9 \pi}\right. \\
& \left.\left.\cos 2 \psi-\frac{\mu a^{2}}{\pi} \cos 3 \psi\right)\right]
\end{aligned}
$$

where

$$
\begin{aligned}
& \dot{a}=-\frac{4 w \mu}{3 \pi} a^{2} \epsilon+a^{2}\left[\frac{14 \mu c_{1} c_{2}}{15 \pi w^{3}}-\frac{4 \mu c_{2}}{9 \pi w} a\right] \epsilon^{2}, \\
& \dot{\psi}=w-\frac{c_{1} c_{2}}{w^{3}} \epsilon+\left[\frac{4 \mu^{2} w a^{2}}{5 \pi^{2}}+c_{1} c_{2}^{2}\left(\frac{2 a}{3 w^{5}}-\frac{c_{1}}{2 w^{7}}\right)\right] \epsilon^{2} .
\end{aligned}
$$

Figure 4 shows a comparison of the analytical solutions for the inclined, curved and the horizontal sections, Eqs. (15)(19) and Eqs. (43)-(45), with the numerical results, Eqs. (13) 


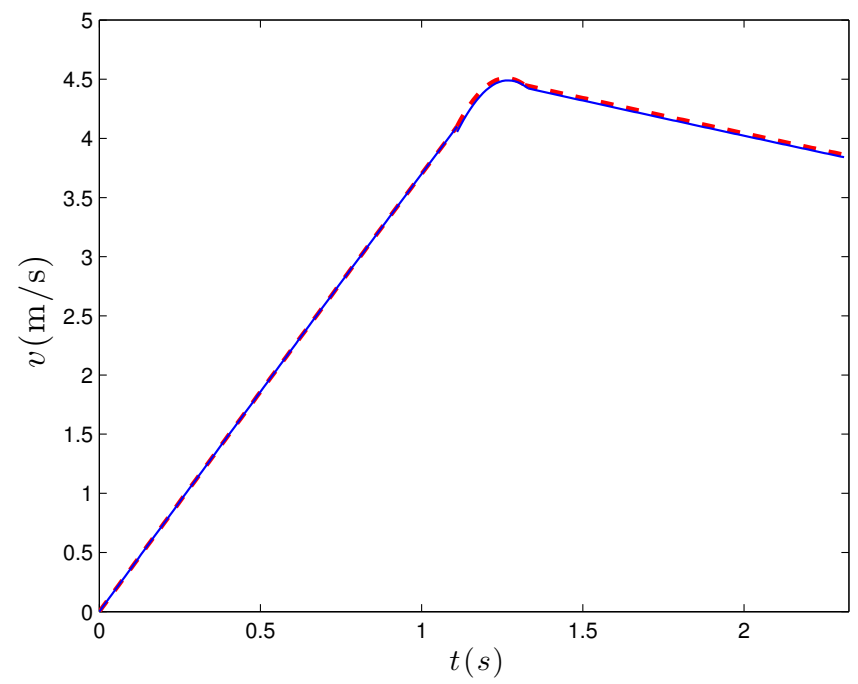

Figure 4. Comparison of the analytical solutions (solid line) for the inclined, curved, and horizontal sections of the track with the numerical simulation (dashed line).

Table 2. Parameters used for the analysis of a prototypical pinewood derby car.

\begin{tabular}{ll}
\hline Parameter & Value \\
\hline$\theta$ & $26^{\circ}$ \\
$d$ & $1.17(\mathrm{~m})$ \\
$\bar{x}$ & $0.098(\mathrm{~m})$ \\
$\bar{y}$ & $0.013(\mathrm{~m})$ \\
$N$ & 4 \\
$g$ & $9.81\left(\mathrm{~m} \mathrm{~s}^{-2}\right)$ \\
$m$ & $0.191\left(\mathrm{Kg}^{-2}\right.$ \\
$m_{o}$ & $2.65 \times 10^{-3}(\mathrm{Kg})$ \\
$r_{o}$ & $0.0151(\mathrm{~m})$ \\
$r_{i}$ & $2.54 \times 10^{-3}(\mathrm{~m})$ \\
$k$ & $0.0107(\mathrm{~m})$ \\
$\ell_{f}$ & $4.21(\mathrm{~m})$ \\
$\ell_{s}$ & $2.16(\mathrm{~m})$ \\
$\beta_{1}$ & 26 \\
$\beta_{o}$ & 34.3 \\
$\mu_{\mathrm{k}}$ & 0.167 \\
$C_{\mathrm{D}}$ & 1.1 \\
$A$ & 0.00146 \\
$\rho_{\mathrm{a}}$ & 1.2041 \\
$I_{w}$ & $3.06 \times 10^{-7}\left(\mathrm{Kg} \mathrm{m}^{-2}\right)$ \\
$I_{G}$ & $2.5205 \times 10^{-4}\left(\mathrm{Kg} \mathrm{m}^{-2}\right)$ \\
\hline
\end{tabular}

and (27), for the parameters given in Table 2 and for $\epsilon=0.01$. From the figure it is clear that the analytical and numerical results are in agreement. In the next section, the derived theoretical results are used to study trends in the velocity and race time for hypothetical changes in the car's physical parameters.
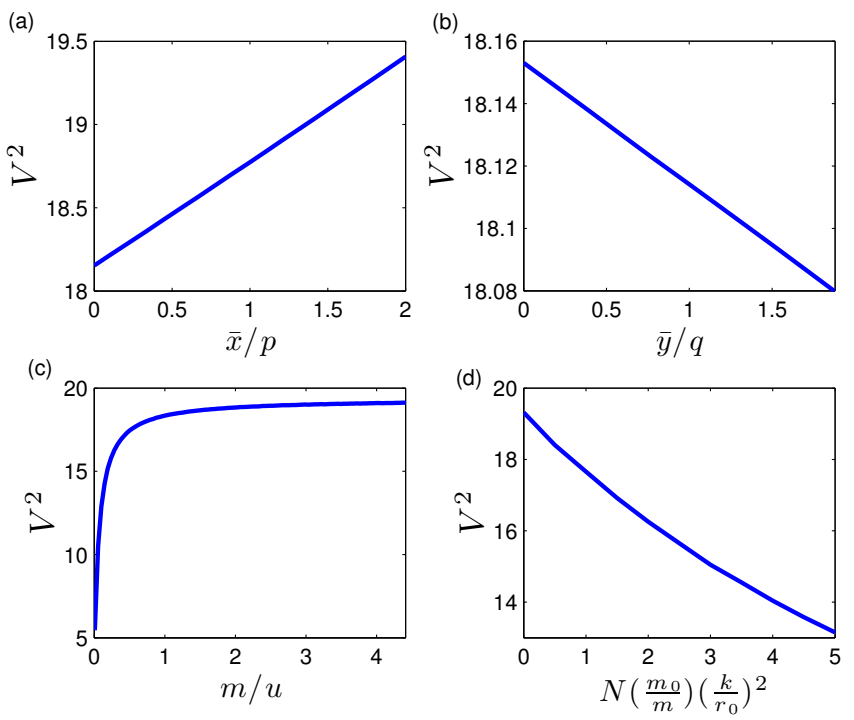

Figure 5. Theoretical trends in $v^{2}$ with the dissipative forces included; results show the effects of varying $\bar{x}(\mathbf{a}), \bar{y}(\mathbf{b}), m(\mathbf{c})$, and the wheel parameters (d) with parameters given in Table 2 and the normalization constants $p=0.09 \mathrm{~m}, q=0.013 \mathrm{~m}$, and $u=0.1091 \mathrm{Kg}$.

\subsection{Trend studies}

A conservative energy balance was used in Sect. 2 to explore trends in the car's peak velocity as key parameters, or groups of parameters, were changed. In this section, we have included the dissipative forces that appear in the governing equations, see Eqs. (13) and (25), to generate trend studies and highlight some additional behavior of interest. While the studies shown in this section were obtained from numerical simulation, we have also validated their accuracy with the analytical solutions, as shown previously in Fig. 4.

The first series of results are shown in Fig. 5. It is interesting that the trends of Fig. 5 are very similar to those presented previously in Fig. 2, results that were obtained by ignoring the dissipation. For example, trends in $v^{2}$ due to changes in $\bar{x}, \bar{y}$, and the wheel parameters are nearly identical. Similarly, both figures show regions where $v^{2}$ can dramatically increase for changes in $m$ and other regions where the peak velocity changes very little for increases in $m$. However, the results from Figs. 2 and 5 are not identical and certain important differences do appear. In particular, the dissipative forces are shown to significantly decrease the peak velocity.

Figure 6 further examines the effect of increasing mass with plots of the car displacement and velocity. It is shown that the car with highest mass is the fastest one, i.e. the first one to reach the end of the track, see Fig. 6a-b. While the peak velocity has already been shown to increase with mass in Fig. 5, the additional insight from Fig. 6 is that the velocity is also larger on other sections of the track. It should be noted that we have chosen two substantially different mass values for the purposes of illustration, i.e. this causes more 


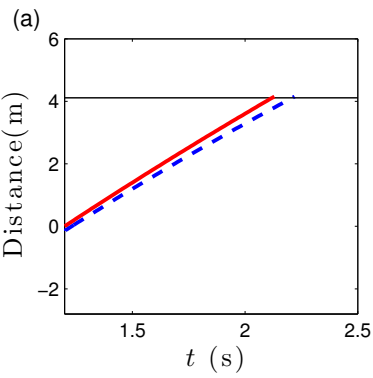

(c)

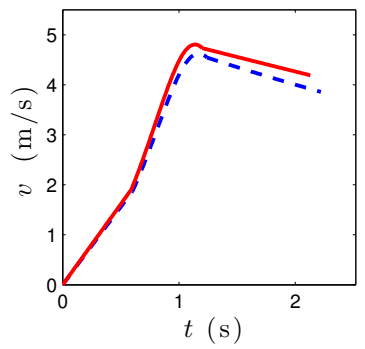

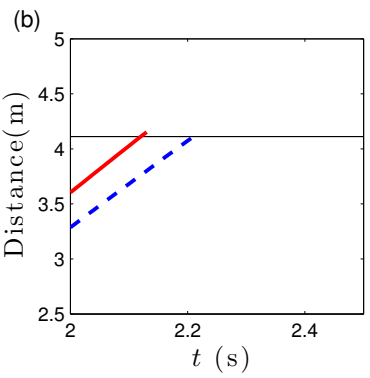

(d)

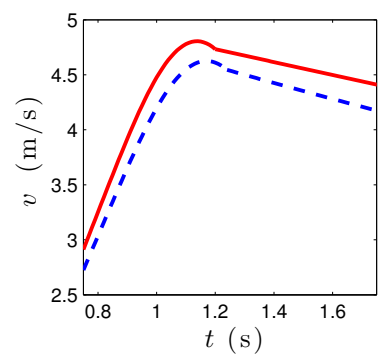

Figure 6. Displacement and velocity plots for two values of the car mass $m=0.05 \mathrm{Kg}$ (dashed), $m=0.35 \mathrm{Kg}$ (solid) and with the remaining parameters given in Table 2. The horizontal line in (a) and (b) represents the finish line. The car with highest mass is the first one to reach the end of the track.
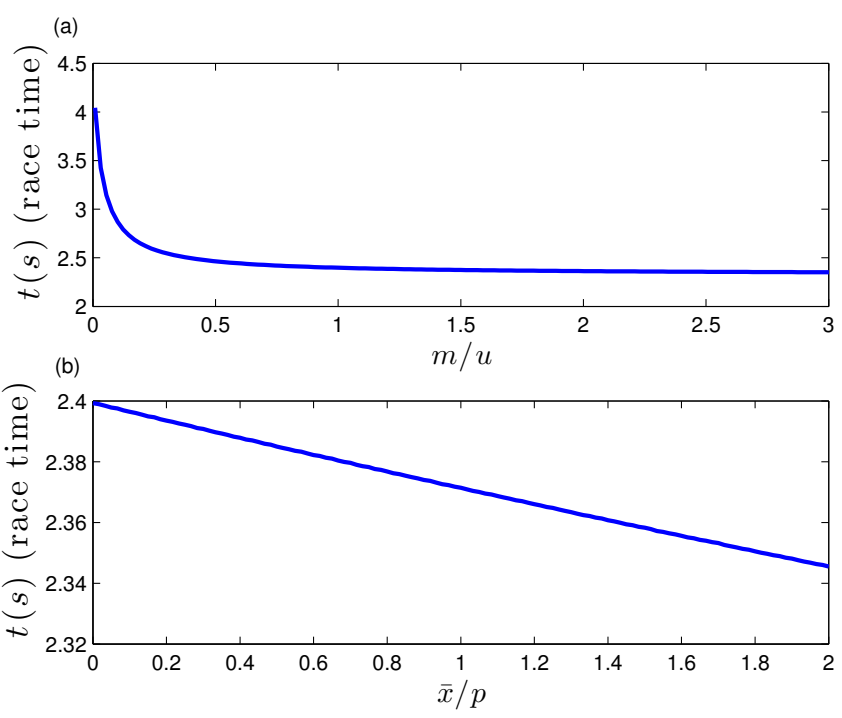

Figure 7. The race time of the car as a function of the car mass (a) and the $\mathrm{x}$-direction location for the car center of mass (b) with parameters given in Table 2 and the normalization constants $p=$ $0.09 \mathrm{~m}$, and $u=0.1091 \mathrm{Kg}$. Beyond a certain mass value the race time barely changes whit increased mass (a).

noticeable differences in the distance and the velocity time histories shown in Fig. 6. Since the race time and not the displacement and velocity is the typical quantity of interest, Fig. 7 shows the trends in race time for variations in the car mass and $\bar{x}$. Focusing on the results of Fig. 7a, one can see regions where altering the mass can either make a large or

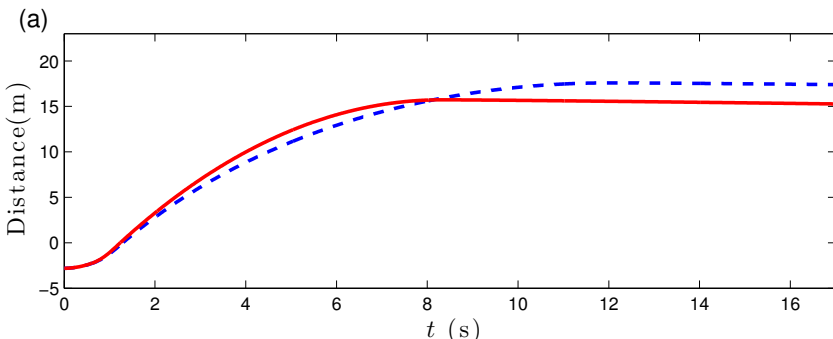

(b)

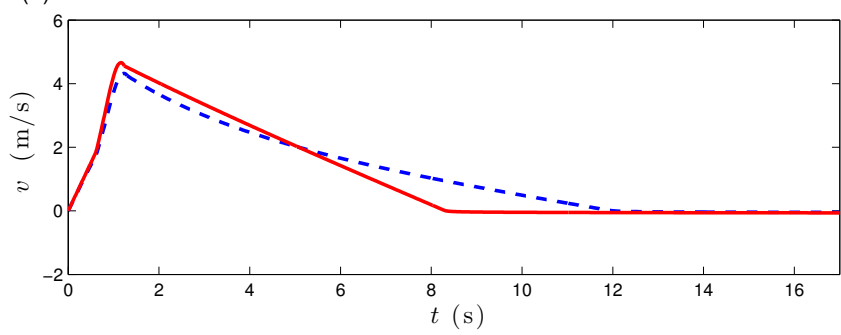

Figure 8. Displacement and velocity for two values of the car mass $m=0.02 \mathrm{Kg}$ (dashed), $m=0.2 \mathrm{Kg}$ (solid) and with parameters given in Table 2 and $\mu_{\mathrm{k}}=0.2$. The heavier (solid) car stops before the lighter car when the track is sufficiently long.

nearly insignificant difference on the race time. In contrast, the results of Fig. $7 \mathrm{~b}$ show the race time only changes linearly with $\bar{x}$.

Given the evidence presented thus far, it might seem reasonable to conclude a car with a larger mass will always finish the first. However, this is not the case and one example where this is not the case is shown in Fig. 8. For this example, the heavier car actually stops before the lighter car when the track is sufficiently long. While this might seem like an obvious case, since the heavier car stopped, other cases also exist where neither car stops, as shown in Fig. 9a. These trends were further explored by generating the 3-D plot of the race time as function of both the mass and the friction coefficient, shown in Fig. 9b, and it can be seen that as $\mu_{\mathrm{k}}$ becomes larger the race time increases with the mass, as opposed to decreasing as originally expected. This is significant because the conservative energy balance analyses completely misses this behavior. The disadvantages of adding mass to the car are only revealed when using the full set of differential equations.

\subsection{Sensitivity analysis}

For nearly any design exercise it is important to consider which factors or design parameters influence performance the most. To gain insight into this question, we performed a sensitivity analysis on the car race time as a function of several key parameters. In the results that follow, we show results from a normalized sensitivity analysis and plots of the UMFs (uncertainty magnification factors), which were determined analytically (Coleman and Steele, 1999; Frey and Patil; Hamby, 1994). The general form for the $i$-th UMF 

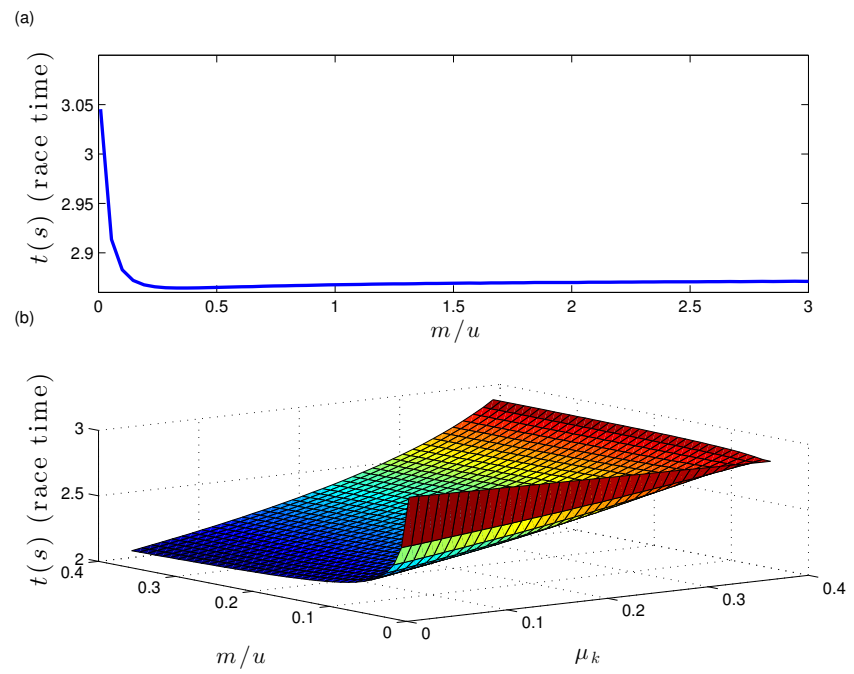

Figure 9. The race time of the car as a function of the car mass with parameters given in Table 2 and $\mu_{\mathrm{k}}=0.35$ (a). The race time of the car as a function of both the car mass and the coefficient friction $\mu_{\mathrm{k}}$ with parameters given in Table 2 and the normalization constants $u=0.1091 \mathrm{Kg}$ (b).

factor can be expressed as follows:

$\mathrm{UMF}_{i}=\frac{X_{i}}{t} \frac{\partial t}{\partial X_{i}}$

where the sensitivity coefficient $\frac{\partial t}{\partial X_{i}}$ is the ratio of the change in output, in our case the race time, to the change in input while all other parameters are held fixed. The quotient $\frac{X_{i}}{t}$ is introduced to normalize the coefficient by removing the affects of units. Therefore a large value of UMF for a certain parameter means that the parameter has more influence on the race time than a parameter with a small UMF value. In Fig. 10, the UMF factor for different parameters is shown for the case of the inclined track, Eqs. (12a), (12b) and (17b). We consider five parameters, namely the projected area of the car $A$, the total car mass $m$, the kinetic coefficient of friction $\mu_{\mathrm{k}}$, the wheel radius $r_{o}$, and the inner radius $r_{i}$. In Fig. 10 the parameters $X_{i}$ are divided by the parameters considered in our experiment and it can be seen that the dependence of UMF on the parameters $A, \mu_{\mathrm{k}}$ and $r_{i}$ is linear while it is nonlinear in the case of $m$ and $r_{0}$. Also, it can be deduced from the figure that overall the radius of the wheel $r_{o}$ has more influence on the race time compared to the other parameters, while the projected area of the car $A$ has less influence. For the parameters considered in our experiment, the race time is sensitive in a decreasing order to $r_{o}, \mu_{\mathrm{k}}, r_{i}, m$, and $A$.

In the next section, experimental investigations are performed and compared to the analytical results.

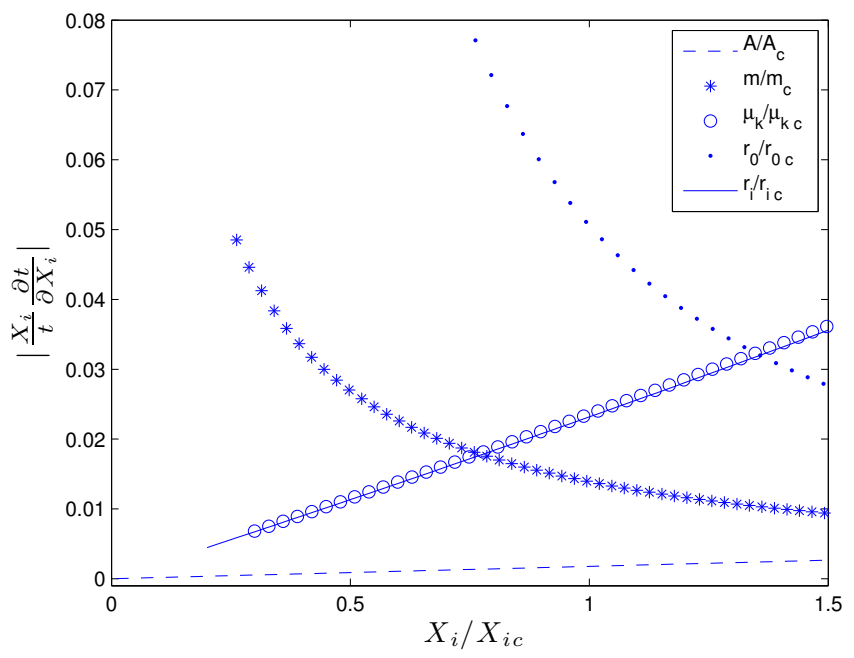

Figure 10. The uncertainty magnification factor as we change the normalized cross section area (dashed line), mass (stars), friction coefficient (circles), outer wheel radius (dotted) and inner wheel radius (solid line) with parameters given in Table 2.

\section{Experimental investigations}

This section describes the experimental investigations performed to validate the trends described in the earlier theoretical sections of the paper. We first describe the experimental track and vehicles used in the experimental trials then proceed to discussing the parameter identification methods. These sections are followed by a discussion of the trends observed in the experimental trials.

\subsection{Track description}

A picture of the experimental track is shown in Fig. 11. As in the theoretical studies, the geometry for the aluminum track can be divided into three distinct regions; the initial inclined region is connected to a relatively short curved section that is then followed by a final straight section that is horizontal. The gravity car begins the race at the starting position on the inclined section. The car is held in place by a spring-loaded peg that protrudes from the track center and is withdrawn to initiate the race. The car then travels down the inclined, curved, and horizontal sections of the track until it reaches the finish line where an automatic timer records the elapsed time. The timer is triggered at the start of the race by a microswitch attached to the start gate. The finish of the race is determined when the car breaks an infrared beam of light that is shone down from the timer onto the sensors in the track. While the car is traveling down the length of the track it also occasionally makes contact with a raised center portion which acts to recenter the vehicle. 


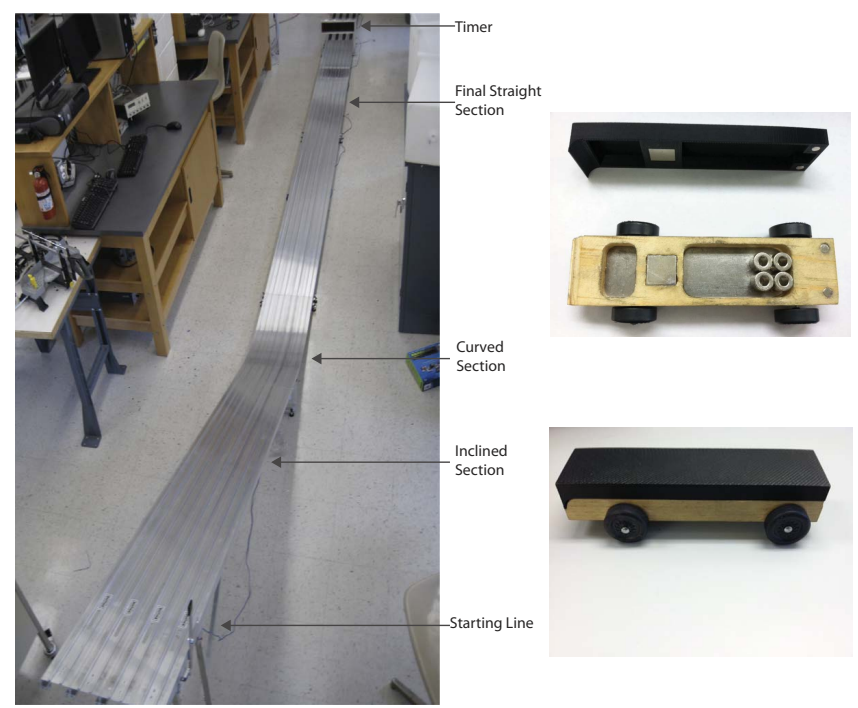

Figure 11. Experimental track and car body used during the experimental investigations. The outer shell of the car is removable to allow for trials with different masses and centers of mass.

\subsection{Gravity car description}

The gravity car is comprised of a base made from a standard pinewood derby kit that has been modified to be fitted magnetically with four plastic shells which differ in shape. The base is hollow and weights can be placed inside to vary the car's center of mass. Four nails act as axles which slide into grooves on the bottom face. A standard base with interchangeable shells was created to isolate the effects of different car bodies on race times by eliminating uncertainty due to variable wheel alignments, friction coefficients, etc. It is common in the pinewood derby to use graphite to reduce friction between the axles and the wheels, but we did not use graphite in our experiment because spills and variability of application would cause inconsistent results.

\subsection{Identification of car parameters}

Several parameters had to be experimentally identified during the course of our investigations. In particular, separate experiments were required to obtain the radius of gyration for the wheels, the mass moment of inertia for the car, and the friction coefficient between each wheel and axle. An instrumented pendulum apparatus was constructed to obtain the vehicle's mass moment of inertia. The procedure consisted of attaching the car to a pendulum platform, measuring the car-pendulum system oscillations, and then using the period between oscillations $T=\frac{2 \pi}{\omega \sqrt{1-\zeta^{2}}}$, where $\zeta$ is the damping ratio obtained via logarithmic decrement and $\omega$ is the linear natural frequency of the car-pendulum system, to extract $\omega$. Using the parallel axis theorem, the mass moment of inertia was obtained from the expression

$I_{G}=\frac{M g \bar{d}}{\omega^{2}}-I_{\mathrm{p}}-m d^{2}$

where $M$ is the total mass of the car and pendulum platform, $d$ is the perpendicular distance from the pivot point to the car center of mass, $\bar{d}$ is the perpendicular distance from the pivot point to the combined car-pendulum center of mass, and $I_{\mathrm{p}}$ is the mass moment of inertia for the pendulum platform. Instrumented wheel spin tests were used to estimate the radius of gyration for the wheels and the wheel-axle friction coefficient. In these tests, the average angular deceleration was determined from time between $n$ complete rotations of the wheel; a laser tachometer was used to detect full tire rotations and monitor the time between successive rotations. If we let $t_{1}$ be a reference time, $t_{n}$ be $n$ revolutions into the future, and $t_{r}$ denote $r$ revolutions into the future, the following expression gives the angular deceleration

$\alpha=\frac{4 \pi}{t_{r}-t_{n}}\left(\frac{r}{t_{r}-t_{1}}-\frac{n}{t_{n}-t_{1}}\right)$,

which can be related to the friction coefficient and tire mass moment of inertia through a summation of forces and moments. More specifically, the following was obtained by summing moments about the axle

$\alpha=-\frac{\mu_{\mathrm{k}} m_{o} g r_{i}}{I_{o}}$.

To solve for $\mu_{\mathrm{k}}$ and $I_{o}$ indenpendently, a special attachment was affixed to the wheels and the spin test was repeated; the second set of experiments that allowed the unknowns $\mu_{\mathrm{k}}$ and $I_{o}$ to be identified.

\subsection{Experimental trends}

Two tests are performed to validate the theoretical trends for car mass and horizontal center of mass discussed in earlier sections. The first holds all of the car parameters constant while varying the car mass. As mass was added between trials, a constant center of mass was maintained by changing the locations of magnetic weights in the hollowed out car body. These tests used the block shell shown at the bottom right of Fig. 11. The second series of tests changed the horizonta 1 center of mass by moving a weight along the length of the car.

The experimental mass plot (a) verifies the theoretical advantage of increasing the car's mass seen in both the energy balance and the numerical simulation of the car's equation of motion, Eqs. (12) and (22). The plot also highlights the diminishing returns of adding mass as the overall mass of the car increases. This trend is exaggerated in the energy balance balance plot (Fig. 2) because it does not take into account the energy lost due to non-conservative forces. The energy balance plot shows the race time levels out at around half of the 

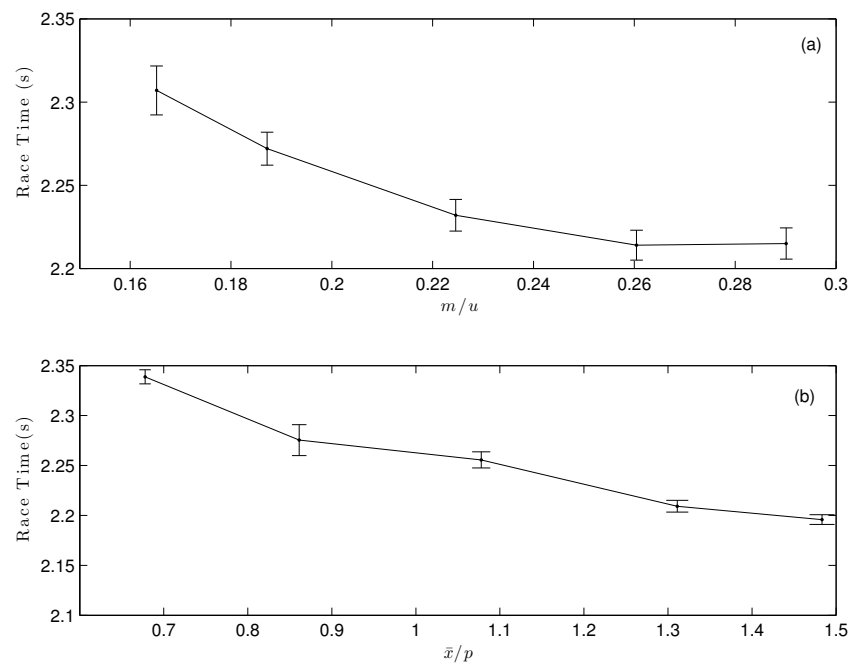

Figure 12. Experimental results of race times for cars with varying mass (a) and varying the horizontal center of mass (b). The mass is normalized by the out-of-the-box car mass of $u=0.1091 \mathrm{~kg}$ and $\bar{x}$ is normalized by the out-of-the-box center of mass $p=0.09 \mathrm{~m}$.

mass of an out-of-the-box car. The plot of race times resulting from the numerical simulation of the car's equations of motion (Fig. 5) portrays a more accurate representation of the relationship between mass and race time. This plot shows the advantage of adding mass starting to level out at around two times the out-of-the-box car mass, which is consistent with the experimental data presented in Fig. 12. As mass is added to the car, it increases the normal force acting on all four wheels, as seen in Eqs. (11a) and (11b). Higher magnitude normal forces correspond to more friction between the axle and the wheel which slows the car down, a phenomena which is not reflected in the energy balance plot. The horizontal center of mass trends found experimentally also comply with both theoretical and simulated results. The simulated results and experimental plots indicate that changing $\bar{x}$ between 1 and 1.5 times the out of the box parameter lowers race times at a faster rate. Increasing $\bar{x}$ moves the center of mass farther from the starting line, which allows the car to continue accelerating along the straight section of the track for longer than a car with a center of mass closer to the front wheels. The advantage gained by a longer acceleration time corresponds to a faster race time. One downside to moving the center of mass to the extremities of the car, one that is not accounted for in the mathematical model, is a higher instance of wheel impacts with the center track partition. Moving the center of mass to either extreme - the front or the rear - causes the car to wobble along the track. It should be noted, however, that in the experimental results, the advantages gained by increasing the center of mass offset the unfavorable wobbling condition.

\section{Discussion}

Experiments showed that increasing the car's mass results in a faster race time, a result that is consistent with theoretical studies of the energy balance and the numerical simulation of the equation of motion. However, when the car's mass exceeds a certain limit, it actually leads to a reduction in race time because of the increased friction caused by a larger mass. This phenomena is not accounted for in the simple energy balance, but comes to light through theoretical investigations. Experimental findings also showed increasing the horizontal center of mass will decrease the race time, a result that validates theoretical findings.

In addition to making the modifications suggested by our theoretical and experimental results, the following techniques can be employed to further reduce race times. Polishing the axles with progression of coarse to fine sandpaper is an effective way to reduce friction and improve race times. Reducing the radius of gyration by removing mass from the outer portion of the wheels is another modification that can effectively reduces race times. If given the freedom to modify the wheel significantly, our sensitivity analysis showed that changing the outer radius of the wheels has one of the largest effects on reducing race time; one could alternatively decrease the inner radius of the wheel or implement a variety of techniques to reduced the friction between the wheels and their axle. Race times were the least sensitive to the cross sectional area of the car. This finding suggests that the significant amount of time and resources put into creating sleek car bodies would be better used to change parameters that the sensitivity analysis found to have a greater impact on race time. If the rules of a given pinewood derby race do not permit wheel alterations, the most efficient parameter to manipulate is the friction between the wheel and the axle.

Another modification that decreases race times involves attaching a long thin object to the front of the car. This technique that takes advantage of the starting mechanism of the racetrack. The car begins to move as soon as the extension loses contact with the retractable pin, which rotates out of the way at the start of the race, essentially giving the modified car a running start. In summary, our findings add to the existing body of knowledge about how to modify gravity cars to reduce race times and offers a ranked order of importance for these modification based on a sensitivity analysis.

Beyond the specific problem studied herein, we believe some of the research findings are generic and could provide insights in other application areas where the relationship between aerodynamics, gravity, and friction alter performance, e.g. skateboarding, rollerblading, and snowboarding. For example, the energy balance expression could also be used to predict trends in peak velocity for a skateboarder. 
Acknowledgements. The authors thank Patrick McGuire for his help in building the experiment. Partial Support from US National Science Foundation is gratefully acknowledged.

Edited by: M. Teodorescu

Reviewed by: M. Teodorescu and one anonymous referee

\section{References}

Chapman, W. L., Bahill, A. T., and Wymore, A. W.: Engineering Modeling and Design, CRC Press, Boca Raton, 1992.

Coleman, H. W. and Steele, W. G.: Experimentation and Uncertainty for Engineers, 2nd Edn., New York, Wiley, 1999.

Coletta, V. P. and Evans, J.: Analysis of a model race car, Am. J. Phys., 76, 903-907, 2008.

Cowley, E. R.: Pinewood Derby Physics, Phys. Teach., 27, 610-612, 1989.

Frey, H. C. and Patil, S. R.: Identification and review of sensitivity analysis methods, Risk Anal., 22, 553-578, 2002.

Garguilo, J. and Garguilo, S.: Winning Pinewood Derby Secrets, Pinewood Pro, 2011.

Hamby, D. M.: A Review of the techniques for parameter sensitivity analysis of environmental models, Environ. Monit. Assess., 32, 135-154, 1994.
Jobe, J. D.: Physics of the Pinewood Derby With Engineering Applications, Missouri City, TX, Self-publishing, 2004.

Meade, D.: Pinewood Derby Speed Secrets, Fox Chapel Publishing Company, Inc., 2006.

Mickens, E. R.: Oscillations in planar dynamic systems, World Scientific, 1996

Minorsky, N.: Nonlinear Oscillations, Van Nostrand, Princeton, N.J., 1962.

Pedigo, T. L.: How to Win a Pinewood Car Derby, Winning Edge Publications, Inc., 2002.

Reinke, P.: Pinewood Winning by the Rules, Eloquent Books, 2010.

Solzak, T. A. and Polycarpou, A. A.: Engineering outreach to cub scouts with hands-on activities pertaining to the pinewood derby car race, Int. J. Eng. Educ., 22, 1077-1096, 2006.

Torvi, D. A.: Using Pine Wood Derby Cars to Introduce Mechanical Engineering to Students in a First Year General Engineering Design Course, 4th Canadian Design Engineering Network (CDEN)/Canadian Congress on Engineering Education (C2E2) Conference, Winnipeg, MB, 22-24 July, 2007. 